\title{
Un análisis exploratorio sobre las experiencias de viajar en el subterráneo de Buenos Aires
}

\author{
Maite Beramendi ${ }^{1}$ y María de los Ángeles Vanesa Romero Gianotti ${ }^{2}$
}

\begin{abstract}
RESUMEN
Las condiciones del viaje influyen en el bienestar de las personas. Esto cobra relevancia considerando que viajar es uno de los diez comportamientos más rutinarios de la vida. El objetivo de este trabajo es analizar las experiencias de los pasajeros en el subterráneo de Buenos Aires en relación con las condiciones de calidad y servicio que perciben que el transporte público les brinda. Para ello se diseñó un estudio exploratorio y se recolectó la información a través de grupos focales. Los resultados indican que los participantes eligen principalmente el subterráneo por su rapidez; y la mayor desventaja se asocia al aglomeramiento de pasajeros. Los participantes no solo priorizan el tiempo de viaje sino trasladarse lo más tranquilamente posible para disminuir los posibles factores de estrés.

Palabras clave: aglomeramiento, bienestar, cambios, rapidez, subterráneo.
\end{abstract}

\section{An exploratory analysis on the experiences of traveling in the underground of Buenos Aires}

\begin{abstract}
The conditions of travelling influence the well-being of people. This becomes relevant considering that traveling is one of the ten most routine behaviors in life. The objective of this work is to analyze the experiences of passengers in the Buenos Aires subway in relation to quality and service conditions perceived by public transport. For this purpose, an exploratory study was designed, and the information was collected through focus groups. The results indicate that participants mainly choose the subway because of its speed; and the greatest disadvantage is associated with crowding of passengers. Participants not only prioritize the travel time but also to be able to travel as calmly as possible to reduce possible stressors.
\end{abstract}

Keywords: changes, crowing, rapid, underground, well-being.

Los sistemas de transporte público son servicios esenciales y necesarios para asegurar la sostenibilidad, la equidad y la habitabilidad de las ciudades (Diab, van Lierop, \& El-Geneidy, 2017). Durante el siglo XX, afloraron las inversiones en el desarrollo de un sistema de subterráneo (e.g., metro) en las grandes ciudades del mundo (Li, Yang, Qin, \& Chonabayashi, 2016), ya que es considerado uno de los medios de transporte más idóneo para atender a los problemas que genera la congestión del tráfico, la polución, los ruidos, los

\footnotetext{
1 Universidad de Buenos Aires, Universidad de la Marina Mercante, Universidad de la Defensa Nacional, Argentina; maiteberamendi@gmail.com; http://orcid.org/0000-0002-6113-5529

2 Universidad de la Marina Mercante, Argentina; marangeles100@gmail.com; http://orcid.org/0000-00032005-8608
} 
tiempos de movilidad de los pasajeros; y porque permite transportar un caudal importante de personas con un bajo coste energético (Jain, Aggarwal, Kumar, Singhal, \& Sharma, 2014).

Además, se convirtió en el transporte preferido por su eficacia y fiabilidad. Bell y Iida (1997) afirman que una red de transporte es confiable cuando es alta la probabilidad de que el sistema pueda cumplir su función original dentro de las condiciones y el tiempo pautados. En este sentido, el subterráneo, a diferencia de los autos o los ómnibus, tiene una menor probabilidad de que ciertos factores (e.g., retrasos, congestiones, accidentes o factores meteorológicos) puedan afectar su correcto funcionamiento. Por todas estas razones, los subterráneos se han convertido en un elemento importante para el desarrollo urbano, económico y un símbolo de las metrópolis, a pesar de su reciente incorporación a las ciudades en términos históricos.

\section{El subterráneo como un medio de transporte que reconstruyó la estructura y dinámica de las ciudades}

Desde la construcción del primer subterráneo en 1863 en Londres, este medio de transporte tuvo como propósito resolver problemas de hacinamiento y enfermedades que pululaban en la ciudad. El subterráneo fue un medio barato y rápido para transportar a trabajadores a los suburbios, los cuales brindaban una mejor calidad de vida (Bradley, 2007, como se citó en Siu \& Zhao, 2013).

En la actualidad, la función del subterráneo sigue siendo la de transportar rápidamente y descentralizar la gran población de las ciudades para poblar los suburbios y disminuir el tránsito automovilístico. Sin embargo, los subterráneos se han convertido en estaciones de servicio (e.g., venta de comida, ropa, etc.), en puntos de referencia de la ciudad y mapas de las zonas más importantes de las ciudades (Siu \& Zhao, 2013).

La red del subterráneo forma un mapa de la ciudad que demarca los espacios socialmente relevantes. Se diseña la planificación de las líneas y estaciones del subterráneo a partir de seleccionar lugares estratégicos o relevantes de la ciudad a los cuales se desea que las personas accedan. En ciertas situaciones, el lugar de interés antecede a la creación del subterráneo, y en otras, la extensión de él revaloriza barrios y espacios públicos. Esto se observa más claramente en las ciudades como, por ejemplo, Londres, Nueva York o Tokio donde el subterráneo recorre una gran extensión de la ciudad (Shuffield, 2017; Siu \& Zhao, 2013).

El mapa del subterráneo proporciona un mapa mental que reestructura la ciudad, distorsionando su espacialidad y sus ritmos. Las distancias recorridas por el metro son percibidas como más cortas que las distancias que las personas caminan o transitan por calle, ya sea por la velocidad del transporte o por la falta de referencias visuales. Por otro lado, los mapas físicos configuran una nueva ciudad donde se amplían las zonas cercanas a las estaciones, se reducen gráficamente las zonas más alejadas o menos transitadas y se invisibilizan los espacios de la ciudad a donde el subterráneo no llega (Shuffield, 2017).

\section{Factores que influyen en el uso del subterráneo}

El éxito del sistema de transporte público depende del número de pasajeros que el sistema es capaz de atraer y retener. Ettema et al. (2011) han hallado que los cambios en las condiciones de viaje (e.g., la mejora de los 
servicios de transporte público) influyen en la satisfacción con el viaje, pero también mejoran el bienestar subjetivo de las personas. Esto es sumamente importante considerando que viajar es uno de los 10 comportamientos más habituales y rutinarios en la vida de las personas, junto con comer, trabajar y dormir (Légal, Meyer, Csillik, \& Nicolas, 2016).

Estudios previos confirman que hay varios factores que afectan la calidad del viaje y el bienestar de las personas. Este último, en general, se lo comprende como la evaluación cognitiva y afectiva que los pasajeros realizan sobre el viaje (Ettema et al., 2011). Muchas de estas investigaciones se han realizado considerando a los usuarios de ómnibus y trenes, y hay pocas que se hayan centrado en los pasajeros del subterráneo.

Morris y Guerra (2015) plantean que hay varios estudios que dan cuenta que las personas disfrutan de ciertos aspectos del viaje como el disfrute estético (observar el paisaje), la emoción de moverse a gran velocidad, la satisfacción de la curiosidad, los sentimientos de dominio y control, escapar del estrés del trabajo y el hogar, etc. Sin embargo, hay otros aspectos que hacen que las personas realmente quieran acortar su tiempo de viaje, ya que su prolongación lo convierte en algo más oneroso, les quita tiempo para dedicarlo al ocio o a su familia y aumenta los niveles de estrés y malestar.

Otros factores que condicionan la calidad del viaje de los pasajeros son el tiempo de espera y la imprevisibilidad del servicio. Considerando que una de las principales demandas de los pasajeros hacia el transporte público es reducir el tiempo de su viaje, a la gente le genera malestar esperar (Shen, Xiao, \& Wang, 2016). En la mayoría de los estudios sobre el tiempo de espera, los resultados indican que las personas vivencian emociones de enojo e incertidumbre (Friman, 2010). Generalmente esto sucede porque observan una incongruencia entre el servicio que desean y el que reciben (Maister, 1985). Además, Maister (1985) afirma que los tiempos de espera generan ansiedad a las personas, y que esta aumenta cuando las personas no saben cuánto es el tiempo real que tienen que esperar. Es decir, cuando el sistema de transporte les avisa a los pasajeros los horarios o las demoras en el servicio, sus niveles de ansiedad disminuyen. Además, en el caso particular de los subterráneos y de los trenes, la ansiedad es más alta, porque no hay una fila que demarque el orden de espera, sino que hay una multitud desordenada de la cual no se sabe cómo va a actuar cuando llegue el transporte a la estación (Maister, 1985). Igualmente, Friman (2010) plantea que el tiempo de espera puede volverse menos displacentero si se generan escenarios que entretengan a las personas.

Otro factor que reduce la sensación de comodidad al viajar es el aglomeramiento. Mohd Mahudin, Cox y Griffiths (2012) afirman que, para analizar este fenómeno en los trenes, medio que comparte varias semejanzas con el subterráneo, se deben contemplar dos dimensiones, una de ellas centrada en (a) la densidad y el espacio disponible, que representan el componente objetivo, y la otra en (b) la percepción del espacio disponible y el número de personas presentes, que caracterizan el componente subjetivo. En su investigación, los participantes evaluaron la densidad física como algo denso, desordenado, confinante, caótico, perturbador y desagradable; así mismo, describieron el ambiente físico como caliente, ruidoso, congestionado y maloliente. En general, las investigaciones han dado cuenta de que el calor, el olor y la incomodidad están asociados con la sensación de hacinamiento y pueden contribuir al comportamiento agresivo y a respuestas interpersonales 
negativas (Mohd Mahudin et al., 2012). En relación con el componente subjetivo, las personas experimentan situaciones donde se sienten que se restringe su comportamiento y aumenta los niveles de estrés. Continuando con esta línea de investigación, Cox, Houdmont y Griffiths (2006) han hallado que la percepción de hacinamiento está vinculada a la limitación, la distracción, el malestar y el estrés. Asimismo, Evans y Wener (2007) han observado la percepción de la invasión del espacio personal, sobre todo, cuando esta acción procede de personas desconocidas como sucede en los trenes, se asocia a mayores niveles de estrés.

\section{El subterráneo en Argentina}

En la Ciudad de Buenas Aires, el nacimiento del subterráneo fue producto de la necesidad de organizar y estructurar el impacto del crecimiento poblacional de fines del siglo XIX y la diversificación de las actividades productivas de la misma (Nesprias, 2011). El crecimiento explosivo de la ciudad por la inmigración hizo que la población crezca tres veces entre 1887 y 1936, pasando de 400.000 a 1.200 .000 habitantes. Esto generó un colapso, y obligó a las personas a trasladarse a los suburbios. Así surge la necesidad de reestructurar la planificación del transporte público para que las personas puedas trasladarse velozmente dentro y hacia la periferia de la ciudad (Singh, 2013). Además, este nuevo transporte no solo quiso combatir el tráfico de la ciudad, sino también modernizarla como en otras ciudades europeas (Nesprias, 2011).

Transcurrieron varios años hasta que, finalmente, se aprobó la construcción y explotación del subterráneo, lo cual ocurrió el 21 de diciembre de 1909. El proyecto aprobado permitió la construcción de lo que hoy se conoce como la Línea A, la primera línea de Iberoamérica y la número 13 en el mundo (Singh, 2014). Esta linea fue inaugurada con 8 estaciones (Plaza de Mayo-Plaza Miserere) el 1 de diciembre de 1913 por el presidente Victorino de la Plaza. Al dia siguiente, 170.000 personas viajaron en el nuevo transporte porteño. En la actualidad, el sistema de subterráneo cuenta con 103 estaciones, que recorren una distancia de $56.9 \mathrm{~km}$ (sin contar el premetro) y traslada diariamente a 1.230.000 personas (Subterráneos de Buenos Aires Sociedad del Estado, 2017).

\section{Los problemas del subterráneo de Buenos Aires}

En la actualidad, el subterráneo de Buenos Aires está llevando a cabo un plan de ampliación que abarca la extensión de la red, los andenes y la mejora general de los servicios como son: la incorporación de locales, cámaras de seguridad y policias, servicio de internet gratuito, aplicaciones de internet para informar los horarios de circulación del subterráneo (Subterráneos de Buenos Aires Sociedad del Estado, 2017)

Una encuesta realizada por el Centro de Estudios Nuevo Milenio (2016) halló que los residentes de la Ciudad de Buenos Aires creen que el subte es el mejor servicio que funciona en la ciudad, aunque presenta problemas relacionados con la limpieza y la infraestructura. Además, a los encuestados les preocupa la falta de seguridad.

Por otro lado, diversas fuentes periodísticas (e.g., Infobae, 2104; Novillo, 2015) dan cuenta de que las críticas más negativas hacia el sistema de subterráneo se concentran en su baja frecuencia y la cantidad de personas que transportan. Siguiendo con esta crítica, Saravia, presidente del Consejo 
Económico y Social de la Ciudad, asegura que el principal problema radica en que hay más pasajeros que cantidad de vagones para trasladarlos, al menos con calidad (Girabsas, 2016).

Otra crítica que se le realiza al subterráneo es que, a pesar de su pronta aparición, su crecimiento no fue continuo en el tiempo. Si se lo compara con los subterráneos más grandes del mundo (e.g., Korea, Pekin, Shangay, Londres, Nueva York) el porcentaje de kilómetros y lineas es pequeño. Saravia, resalta que hace 40 ó 50 años atrás la red de subtes porteña era comparable a la de Madrid o la de París, pero hoy en día es casi el $10 \%$ de esas redes debido a que la extensión del subterráneo no ha sido ampliada (Girabsas, 2016).

En el marco de lo expuesto, se observa que viajar en el subterráneo de Buenos Aires implica ciertos problemas para los usuarios, pero no hay investigaciones previas que los analicen de manera sistemática. Como se comentó anteriormente, el mal funcionamiento de los transportes afecta la calidad de vida de las personas y su bienestar. Por eso, es relevante llevar a cabo un primer estudio que analice la calidad y el servicio del subterráneo para comprender los factores positivos y negativos que perciben los usuarios, y que estos últimos sirvan como diagnóstico para pensar futuras mejoras en la calidad del servicio de este transporte público.

\section{MÉTODO}

\section{Diseño y tipo de estudio}

Se diseñó un estudio no experimental, transversal y exploratorio. Para el análisis de la información obtenida en los grupos de discusión se contempló el modelo de diseño denominado emergente-sistemático de grupos focales (Onwuegbuzie, Dickinson, Leech, \& Zoran, 2011). Onwuegbuzie et al. (2011) proponen llevar a cabo múltiples grupos focales con el fin de corroborar si la información que surge en uno se corrobora en otro, para poder garantizar la saturación de los datos y/o teórica. Los autores denominaron a este diseño emergente-sistemático, porque lo emergente refiere a la información que surge en un grupo, mientras que lo sistemático se refiere a la información que surge en otro grupo y verifica la información previa (e.g., Onwuegbuzie et al., 2011). Este diseño se complementa con la técnica de análisis de contenido. Esta técnica sistematiza discursos con el propósito de ordenar y analizar su contenido manifiesto para crear categorias de análisis, y así para poder realizar deducciones reproducibles y aplicables a un contexto particular (Fernández Chaves, 2002).

\section{Técnicas y herramientas de recolección de información}

Se utilizó la técnica de grupo de discusión. Esta técnica de recolección de información consiste en reuniones grupales, en las cuales los participantes conversan sobre varios temas en un ambiente relajado e informal (Dominguez Sánchez-Pinilla \& Davila Legerén, 2008).

Esta técnica focaliza de manera estratégica en aquellos elementos y preguntas que inducen a los participantes a hablar y a interactuar entre ellos, y que son relevantes para la investigación (Dominguez Sánchez-Pinilla \& Davila Legerén, 2008). Por ello, para la conducción de las sesiones de grupos focales se consideró una guía de temas de discusión con preguntas semiestructuradas, que permitieron orientar la discusión. Sin embargo, como 
sugiere la técnica de grupos focales, se realizaron nuevas preguntas a partir de las temáticas emergentes, ya que el grupo focal tiene como uno de sus supuestos básicos que la información se construye a partir de la interacción (Heritage, 2004).

Para estructurar las discusiones grupales se construyó un guía que incluyó una breve introducción y siete preguntas orientadoras que se detallan a continuación. De este conjunto de preguntas, se utilizaron tres de ellas para realizar este estudio: la a, b y g.

\begin{abstract}
Introducción: Como ya les comenté brevemente en la invitación, la idea de armar este grupo es discutir un poco sobre la experiencia de viajar en el subterráneo de Buenos Aires. Por lo tanto, para empezar, y para entrar un poco en confianza querría preguntarles: (a) ¿Cuáles son las ventajas del subte que hacen que lo elijan como medio de transporte?; (b) ¿Cuáles son las desventajas del subte como medio de transporte?; (c) Pensando en el subte ¿Cómo creen que se relacionan las personas allí, es decir, qué dinámica observan entre los pasajeros?; (d) ¿Cuáles son las normas de seguridad y convivencia que conocen que posee el subte?; (e) ¿Cuáles se cumplen?; (f) ¿Cuáles se incumplen?; y (g) ¿Algún otro comentario que quieran hacer y que no fue abordado?
\end{abstract}

Para recolectar más información sobre los participantes, se les aplicó un cuestionario auto-administrado que contenía el consentimiento informado y las siguientes preguntas: género, edad, frecuencia con la cual utiliza el subterráneo, el nivel educativo alcanzado, auto-percepción de clase y posicionamiento ideológico.

\title{
Participantes
}

La muestra fue intencional y se utilizó la técnica de bola de nieve para poder llegar a un mayor número de participantes. Esta estuvo compuesta por 21 usuarios argentinos del subterráneo de Buenos Aires. El promedio de edad fue de 44 años $(D E=16.12 ;$ Mín= 20; Máx=70). En la tabla 1 se describen los datos socio-demográficos de la muestra total y de cada grupo focal en particular.

\section{Procedimiento}

Se realizaron cuatro grupos focales que fueron diseñados siguiendo el criterio de máxima variabilidad. Los grupos se realizaron en una universidad de la Ciudad de Buenos Aires. Los participantes fueron invitados a través de la universidad, de conocidos y por medios digitales. Las reuniones se llevaron en un ambiente relajado y distendido, aislado de cualquier tipo de interrupción y se los invitó con bebidas calientes y masas.

Las actividades tuvieron una duración entre 60 y 90 minutos. Las dinámicas grupales fueron grabadas y se tomaron notas de estas. Los participantes respondieron un cuestionario auto-administrado. 
Tabla 1.

Datos sociodemográficos de la muestra por grupos y total.

\begin{tabular}{|c|c|c|c|c|c|}
\hline & $\begin{array}{c}\text { Grupo } 1 \\
n=5\end{array}$ & $\begin{array}{c}\text { Grupo } 2 \\
n=6\end{array}$ & $\begin{array}{c}\text { Grupo } 3 \\
n=4\end{array}$ & $\begin{array}{c}\text { Grupo } 4 \\
n=6\end{array}$ & $\begin{array}{l}\text { Total } \\
n=21\end{array}$ \\
\hline \multicolumn{6}{|l|}{ Género \% } \\
\hline Femenino & 50 & 66.7 & 50 & 66.7 & 61.9 \\
\hline Masculino & 50 & 33.3 & 50 & 33.3 & 38.1 \\
\hline $\begin{array}{l}\text { Edad } \\
\qquad M(\mathrm{DE})\end{array}$ & $55(14.47)$ & $40.50(9.31)$ & $50.50(19.77)$ & $34(16.14)$ & $44(16.12)$ \\
\hline \multicolumn{6}{|c|}{ Frecuencia de uso del subterráneo \% } \\
\hline Varias veces por semana & 60 & 83.3 & 75 & 66.7 & 72.7 \\
\hline Una vez por semana & 20 & 16.7 & 25 & 16.7 & 18.2 \\
\hline Una vez cada 15 días & 20 & 0 & 0 & 16.7 & 9.1 \\
\hline \multicolumn{6}{|l|}{ Nivel educativo alcanzado \% } \\
\hline Secundario incompleto & 0 & 0 & 0 & 16.7 & 4.8 \\
\hline Terciario incompleto & 0 & 16.7 & 25 & 0 & 9.5 \\
\hline Terciario completo & 0 & 0 & 25 & 0 & 4.8 \\
\hline Universitario incompleto & 20 & 66.7 & 50 & 66.7 & 42.9 \\
\hline Universitario Completo & 20 & 16.7 & 0 & 16.7 & 23.8 \\
\hline Posgrado completo & 60 & 0 & 0 & 0 & 14.3 \\
\hline \multicolumn{6}{|l|}{ Auto-percepción de clase \% } \\
\hline Clase Media-Baja & 0 & 0 & 25 & 0 & 4.8 \\
\hline Clase Media & 0 & 50 & 0 & 33.3 & 23.8 \\
\hline Clase Media-Alta & 100 & 50 & 50 & 66.7 & 66.7 \\
\hline Clase Alta & 0 & 0 & 25 & 0 & 4.8 \\
\hline \multicolumn{6}{|l|}{ Posicionamiento Ideológico \% } \\
\hline Izquierda & 20 & 0 & 25 & 0 & 9.5 \\
\hline Centro & 80 & 100 & 75 & 100 & 90.5 \\
\hline Derecha & 0 & 0 & 0 & 0 & 0 \\
\hline
\end{tabular}

\section{Análisis de los datos}

Para el tratamiento de la información se utilizó el análisis basado en la transcripción (Onwuegbuzie et al., 2011) que fue procesado mediante el programa Atlas $\mathrm{Ti}$, que es un programa computacional que posibilita segmentar los datos en unidades, codificar datos y construir teoría (Muñoz Justicia, 2005). Para el análisis de los datos se utilizó la Teoría Fundamentada (Corbin, 2017). Según Corbin (2017), este método cualitativo es único por cinco razones: (a) permite elaborar una teoria a partir de la recolección de datos, (b) el investigador no trabaja con un marco teórico previo, porque si no, no construye un cuerpo de conocimiento a partir de los datos que recolecta; (c) la recopilación de datos y los análisis de los mismos están relacionados (luego de recoger los datos iniciales, el investigador los analiza y estos forman una base para la posterior recopilación de datos, proceso denominado muestreo teórico); (d) este método no es la sumatoria de recolecciones individuales de casos sino un análisis conjunto para crear conceptos y elaborar una teoría; y (e) el diseño de la investigación puede ir desarrollándose continuamente, ya que no está predelimitado.

Para comenzar el análisis, se releyó reiteradas veces las transcripciones, se procedió al análisis. En primera instancia, se procedió a la codificación abierta; en segunda instancia a la codificación axial, y finalmente se crearon las familias de redes. Sin embargo, como plantean Strauss y Corbin (2002), el proceso de codificación no es lineal, sino que se superponen y entrecruzan los diversos tipos de codificación, pues emergen nuevas formas de comprender el fenómeno y conlleva a reestructurar el modelo planteado. 
Para analizar los datos extraídos del cuestionario se utilizó el programa estadístico SPSS .21, el cual permitió realizar análisis descriptivos.

\section{RESULTADOS}

A partir del análisis de los grupos focales se construyeron 6 familias de categorias: Transporte eficiente, Infraestructura material, Mejoramiento en los últimos años, Falta de comodidad, Falta de autoridad y seguridad y Comparación con otros medios de transporte. A continuación, se describen cada una de ellas con sus subcategorias correspondientes y se exhibe en un cuadro las citas textuales de los participantes (ver Anexo):

1. Transporte eficaz y eficiente: esta familia de categoría abarca las ventajas principales que manifestaron los participantes que se asocian a la rapidez del medio de transporte, la menor cantidad de interrupciones en su servicio, su fácil uso, su bajo costo y su accesibilidad a zonas claves de la ciudad.

1.a. Rapidez: todos los pasajeros manifestaron que utilizan el subterráneo como medio de transporte por su rapidez. Según ellos, en la Ciudad de Buenos Aires el tránsito por tierra está altamente congestionado por la cantidad de automóviles públicos y privados, y esto aumenta el tiempo que le dedican al viajar. Además, explicitaron que la Ciudad de Buenos Aires está viviendo muchos cortes de calles, avenidas y autopistas por protestas, piquetes $^{i}$ y otros eventos, lo cual hace que los recorridos se vean interrumpidos, que las personas no sepan la nueva ruta de su viaje y no puedan calcular los tiempos reales que tardarán para llegar a sus destinos. Esto crea un caos en la ciudad y altera concretamente el flujo del tránsito. En cambio, el subterráneo vivencia menos interrupciones por transitar bajo tierra y no tener que lidiar con estos inconvenientes. Y en el caso de que hubiera un inconveniente en el servicio, la empresa expide certificados en cada estación que dan cuenta de los problemas acaecidos y que pueden ser presentados en las instituciones requeridas.

1.b. Zonas estratégicas: los participantes remarcaron que el subterráneo es clave para transitar ciertas zonas de la ciudad, sobre todo, aquellas zonas que concentran universidad, edificios gubernamentales, entre otros. Especificamente, algunos participantes manifestaron que para acceder al Microcentro de la ciudad el transporte más idóneo es el subterráneo, ya que cualquier otro medio duplica el tiempo del viaje.

1.c. Fácil uso: algunos participantes remarcaron que el subterráneo es un medio de transporte fácil de utilizar, ya que los recorridos son claros y directos. Ellos explican que solo realizando unas combinaciones pueden acceder a gran parte de la ciudad y de manera sencilla. A pesar de que el subte los deje un poco alejados de su destino, prefieren tomarlo y caminar las cuadras faltantes, antes que complejizar su viaje aprendiendo nuevos recorridos o tomando varios transportes. Además, algunos aclaran que no solo lo utilizan en su ciudad de residencia, sino que les gusta tomarlo cuando viajan al exterior. Su sencillez permite que puedan tomarlo fácilmente en cualquier ciudad del mundo.

1.d. Económico: algunos pasajeros de estos grupos focales explicitaron que el viaje es económico, sobre todo, pensando en que los trayectos alcanzan muchos puntos de la ciudad (hay que aclarar que luego de estos grupos 
focales el subterráneo aumentó su preció y superó el costo del viaje en colectivo. Sin embargo, si la gente viaja seguido, diariamente, el costo del subte sigue siendo más barato que el del colectivo).

2. Mejoramiento del subterráneo en los últimos años: en esta categoría se enmarcan los comentarios de los participantes que enumeraron mejoras en el funcionamiento del transporte de estos últimos años y que abarcan diversas aristas.

2.a. Expansión de la red del subterráneo: los participantes plantearon que la red se amplió, ya que se incorporaron varias estaciones en las Líneas A y B, y se construyó la Línea $\mathrm{H}$; así como también se crearon más estaciones que unen las líneas y permiten una mejor movilidad por la red del subterráneo.

2.b. Limpieza: las personas afirmaron que los subterráneos nuevos están más limpios que antes, sobre todo, porque no tienen publicidad, ni pegatinas (e.g., publicidad en versión papel que pegaban los pasajeros para publicitar sus servicios) lo que implica una disminución del ruido visual.

2.c. Menos robos: los participantes remarcaron que disminuyeron por tres razones. En principio, la gente cree que puede ser víctima de un robo en cualquier momento. Esto hace que estén más alerta y aumente el monitoreo de control que ellas realizan de las personas y sus comportamientos. En segundo lugar, hay una mayor comunicación entre los pasajeros. Si una persona ve algún movimiento sospechoso le alerta a otra persona para que no sea víctima de un robo. En tercer lugar, los maquinistas avisan por alto parlante a los pasajeros que cuiden sus pertenencias cuando ven subir a gente sospechosa o "ya conocidos por ellos". Así perciben que hay un mayor control, aunque estas formas de vigilancia son informales y no incluyen a personal especializado en la seguridad ciudadana.

3. Falta de Comodidad: en esta categoría se enmarcan los comentarios de los participantes que aluden a la falta de comodidad de los pasajeros al usar este medio de transporte.

3.a. Aglomeramiento: las personas de los grupos de discusión dijeron que el caudal de personas es alto en el subterráneo. Todos remarcaron que en las horas pico (e.g., hora punta) es muy dificil subirse al transporte, a tal punto, que deben dejar pasar varias unidades para ingresar o prefieren tomar el subterráneo contrario a su trayecto de viaje para poder llegar a una estación cabecera (i.e. estación donde comienza el recorrido), esperar que se vacíe la unidad y comenzar su viaje de origen sentados. Muchos reconocieron que el aglomeramiento de personas aumentó por las extensiones del subterráneo. Además, los participantes sienten que el aglomeramiento genera sensaciones y sentimientos negativos que hacen displacentero el viajar. La gente manifiesta que el calor se hace insoportable, que se está apretado, los espacios personales no son respetados, la gente te apoya, te golpea, te asfixia, y se genera tensión entre los pasajeros.

3.b. Encierro: algunos participantes manifestaron que el viajar en el subterráneo les genera una sensación de sofocación, ya que no hay luz y se visualizan bajo tierra. Creen que existe una mayor dificultad para poder salir ante cualquier accidente y eso también les genera emociones negativas. Además, manifestaron que, por las características del transporte, no hay un paisaje que permita mirar al exterior, entonces las miradas se focalizan en las 
personas y esto produce un encuentro de miradas que puede ser incómodo. Por eso, varios participantes plantean que las personas utilizan los celulares, tablets o libros para focalizar su atención en algo y no incomodarse con miradas ajenas.

3.c. Falta de limpieza: los participantes marcaron que hay una falta de limpieza y mantenimiento del subterráneo, ya sea en andenes y vagones. Según ellos, los pasajeros son descuidados, ya que tiran papeles o descuidan el transporte; y por el otro, la empresa no cuida ni limpia como debería para brindar un buen servicio. Además, los participantes reconocen que la limpieza varía según la línea del subterráneo.

4. Condiciones de infraestructura material del sistema de subterráneos: en esta categoria se reconoce que hay cuestiones estructurales materiales del subterráneo que son positivas y otras negativas. Sin embargo, en general en el foco de los participantes hay una tendencia a remarcar aquellas cuestiones que faltan o que están funcionando mal. En algunos puntos específicos hay una ambivalencia mayor, como, por ejemplo, los nuevos vagones que se están utilizando.

4.a. Frecuencia del medio de transporte: los pasajeros remarcaron que hubo un aumento en la frecuencia del subterráneo, que permitió agilizar los tiempos de viaje de los usuarios, y acortar los tiempos de espera entre cada unidad. Sin embargo, creen que deben recortarse aún más, ya que se alcanzaron tiempos de espera de 5 minutos, pero comparándolos con otros sistemas de subterráneo del mundo siguen siendo altos. Muchos manifestaron que para cambiar los tiempos de espera tienen que haber modificaciones estructurales que permitan la incorporación de más unidades y negociaciones con los gremios para que trabajen más en la misma cantidad de horas.

4.c. Sistemas de acceso: los participantes se quejaron de que las escaleras mecánicas no funcionan frecuentemente en muchas estaciones, y que tardan en arreglarlas. Además, plantearon que las escaleras fijas son angostas para todo el caudal de gente que transporta, sobre todo, cuando no funcionan las escaleras mecánicas. Algunos, también remarcaron la falta de ascensores como un medio que sería realmente útil para personas con discapacidades motoras.

4.d. Andenes: algunos participantes acordaron que los andenes son estrechos, que en la distancia entre la pared y la línea amarilla que demarca la zona de espera, hay pocos metros. Además, manifestaron que hay pocos molinetes que permitan la entrada a los andenes. Ellos plantean que el sistema de pago del subterráneo se modernizó, lo que implicó un cambio en los molinillos. Sin embargo, la compañía no agregó nuevos, sino por el contrario, solo cambió algunos, dejando molinillos del viejo sistema que solo sirven para salir. También dijeron que falta un sistema que señalice donde las puertas de los vagones se abren. Ellos creen que este cambio ayudaría a que haya mejor organización para esperar las unidades, y para ascender y descender.

4.e. Vagones: en principio la gente coincide en que la incorporación de nuevos vagones trajo varias mejoras. Ellos reconocen que las unidades realizan menos ruido por el cambio del sistema de ruedas, y que esto contribuye a que haya menos ruido sonoro. Creen que es un avance que los vagones tengan aire acondicionado, ya que la cantidad de gente y el calor bajo tierra muchas veces hace que sea dificultoso respirar. Con respecto a este 
tema, varios se quejaron de que está mal regulado, y que por ello muchas veces el clima es destemplado, en verano la gente tiene frío, sobre todo, en las horas no pico. Igualmente, el mayor reclamo se centraliza en las pocas unidades que poseen aire acondicionado. En relación con los nuevos vagones, afirmaron que tiene ciertas desventajas, como, por ejemplo, que los asientos son pequeños y que hay menos, y que los pasillos son angostos, que están pensados para personas pequeñas; además, visualizan que en ciertas estaciones se genera un desnivel entre los vagones y el andén; por lo contrario, creen que es una ventaja que estos coches tengan más barandas que le permitan a las personas sujetarse mejor. Además, surgió el tema de los vagones exclusivos para mujeres y para mascotas pequeñas. Las opiniones estuvieron encontradas, ya que muchos dijeron que la exclusividad de un espacio para mujeres no resuelve un problema, sino que es retroceder en el tiempo donde las divisiones entre hombres y mujeres estaban más marcadas. Otros remarcan que es una opción y el que no quiera usarla puede no hacerlo, pero quien quiera viajar de dicha manera lo puede hacer. En relación con la inclusión de los animales en el medio de transporte, las personas estaban desinformadas de las condiciones que debian cumplimentar las mascotas para poder viajar, lo que generó que los participantes piensen que podian tener contacto con ellos cuando en realidad no lo tendrian.

4.f. Comunicación dentro del subterráneo: algunos participantes plantearon que muchas veces la señalización de las estaciones está desincronizada, y que esto trae aparejada confusión y muchos inconvenientes, sobre todo en las horas pico, donde circular por el vagón es complicado. Otros participantes hicieron visible que, ante ciertos inconvenientes, como el cierre de una de las salidas del subterráneo, la compañia no lo anuncia y que las personas llegan al destino y se enfrentan con el problema, cuando si fueron avisados podrian sortearlo de una mejor manera.

4.g. Limitación de la red de subterráneo: algunos participantes dijeron que el sistema de subterráneo es pequeño, ya que muchos puntos de la ciudad no son contemplados en este medio de transporte (e.g., aeropuerto), y algunos hasta desearon que conecte a la Ciudad de Buenos Aires con el Conurbano Bonaerense (e.g., los suburbios de la ciudad). En este sentido, muchos hicieron referencia a que el crecimiento de la ciudad no fue consistente en relación con la ampliación de la red de subterráneo.

5. Falta de seguridad y autoridades: en esta categoría se incluye la sensación de inseguridad que vivencian los pasajeros, porque no hay personal de control o autoridad que regule el comportamiento en el subterráneo, y también por la cantidad de robos que perciben o sufrieron.

5.a. Falta de personas que regulen: algunas personas afirmaron que se sienten inseguras en este medio, porque no hay referentes que controlen y regulen el medio. No hay una figura de autoridad a la cual se pueda acudir ante un problema.

5.b. Robos: todos los participantes manifestaron que participaron de algún robo, ya sea porque lo observaron o porque fueron víctimas de robos en el subterráneo. Creen que esta práctica es cotidiana en este medio, ya que no hay seguridad que vigile y la gente se puede escapar fácilmente.

6. Comparación con otros medios de transportes: todos los participantes reconocieron que el medio de trasporte más rápido es el subterráneo, sobre todo, si se considera la franja horaria diurna, ya que muchos lo prefirieron 
antes que los taxis y autos particulares. Además, dijeron que este medio puede evitar interrupciones por manifestaciones, cortes de calle y problemas de tráfico, lo cual lo convierte en un medio más confiable y preciso. Por otro lado, algunos plantearon que toman colectivos porque el recorrido es mayor y llega a lugares a los que no accede el subte. Además, varios participantes afirmaron que en los colectivos el aglomeramiento de personas es menor, ya que hay una figura, el chofer, que controla el caudal de personas, y cuando no hay más lugar no deja subir a nadie más. En cambio, son recurrentes los comentarios que hacen referencia a la falta de autoridad que controle la seguridad, la venta ambulante o la mendicidad en el subterráneo. Es decir, ante un problema la gente no sabe a quién acudir. Otra desventaja que vivencian las personas en el subterráneo en comparación con el colectivo es el encierro, ya que en estos últimos la gente puede disfrutar de un paisaje, de la luz, del aire. En este sentido, muchos argumentan que esta situación genera que la gente disfrute menos el viaje en subterráneo y solo esté pensando en llegar lo antes posible. En relación con la seguridad, las opiniones de los participantes fueron variadas, ya que algunos se sienten más seguros en el subterráneo y otros en el taxi, pero varias mujeres dijeron preferir tomar cualquiera de esos transportes antes que tomar un taxi, ya que el estar rodeadas de personas les da seguridad. En relación con la comparación con los autos, remarcaron que el subterráneo es rápido, no lidian con el tráfico y no hay que pagar playas de estacionamiento caras. Sin embargo, en zonas no tan transitadas, varios participantes prefieren movilizarse con sus autos particulares, ya que es más cómodo y evitan el aglomeramiento de personas.

\section{DISCUSIÓN}

A partir de los resultados hallados, se llega a la conclusión de que los participantes reconocen ciertas ventajas del subterráneo de Buenos Aires. En primer lugar, lo consideran el medio de transporte público más veloz para viajar por la ciudad, además se incrementa esta ventaja porque evita las congestiones del tránsito y los cortes de calle por protestas. En segundo lugar, remarcan que es un transporte económico. Este hecho se materializa por la subvención que el Gobierno de la Ciudad de Buenos Aires le otorga al sistema de subterráneos. Por ejemplo, si se lo compara con el sistema de Londres, los costos del subterráneo argentino no llegan ni a la décima parte. En tercer lugar, reconocen que el subterráneo es esencial para llegar a lugares nodales de la ciudad como el centro porteño, donde se encuentran los organismos públicos principales, bancos y empresas. Desde hace varios años se prohibió el ingreso de autos particulares a esta zona para organizar el tránsito y promocionar el uso del transporte público y, en este sentido, el subterráneo cumple su función de brindar un mejor acceso a aquellas zonas relevantes de la ciudad y configurar un mapa acorde a las necesidades (Shuffield, 2017; Siu \& Zhao, 2013). En cuarto lugar, manifiestan que hubo mejoras, ya que se amplió la red del subterráneo, los vagones no tienen publicidad, lo que disminuye el ruido visual, y crean una sensación de mayor limpieza, y reconocen que hay mayores señalizaciones que brindan un mejor servicio.

En este sentido, el subterráneo de Buenos Aires logra transportar rápidamente a los pasajeros (Siu \& Zhao, 2013), quienes perciben ciertas mejoras. Esto es concordante con encuestas previas que se llevaron a cabo y politicas nombradas por instituciones gubernamentales (Centro de Estudios 
Nuevo Milenio, 2016; Gobierno de la Ciudad de Buenos Aires, 2017). Sin embargo, la falta de comodidad y ciertos problemas estructurales materiales del subterráneo hacen que la gente dude en usarlo o simplemente elija otros medios de transporte y, además, tienen un impacto negativo en la calidad del viaje de las personas (e.g., sensación de sofocación, asfixia, invasión del espacio personal, clima social negativo, entre otros).

Principalmente, el aglomeramiento de pasajeros, y sus consecuencias, generan un nivel mayor de incomodidad en los participantes al igual que en los hallazgos de las investigaciones sobre trenes realizados por Mohd Mahudin et al. (2012), Cox et al. (2006) y Evans y Wener (2007). Los participantes de este estudio caracterizan al ambiente del subterráneo, sobre todo en horas pico, como algo caótico, perturbador y desagradable; reconocen emociones asociadas al malestar, sobre todo, por sentir que existe una violación de su espacio personal. Esto cobra mayor relevancia en el contexto local, porque por un lado facilita conductas de acoso sexual, a tal punto que se contemplaron tomar medidas como en otros países como México, donde hay vagones especiales para mujeres. En este estudio, algunos de los participantes acordaron que es una buena propuesta designar estos vagones especiales para controlar estas prácticas. Sin embargo, la mayoria de los participantes, y sobre todo las mujeres, se posicionaron en contra de esta postura, ya que creen que perpetúa prácticas sexistas que no atienden al problema primordial que esconde el maltrato de género. Y por el otro lado, el aglomeramiento genera confusión y caos, allanando el terreno para el surgimiento de conductas delictivas. Como muchos participantes manifestaron, ellos ven que el robo es una práctica ordinaria. Todos participaron de un robo, ya sea porque fueron víctimas o porque observaron uno. En este sentido, sienten la falta de una figura de autoridad que organice y regule estos comportamientos, y otros, relacionados con la seguridad de las personas.

Otro factor que disminuye la satisfacción con el viaje es el tiempo de espera y la imprevisibilidad del servicio. En su mayoria, los participantes manifestaron que la frecuencia del servicio debia aumentar como así también avisar a los pasajeros los tiempos reales de espera. Como plantean Friman (2010) y Maister (1985), exhibir los horarios de arribo de los vagones ayudaria a disminuir el enojo y la ansiedad de los pasajeros y promover prácticas positivas para mejorar la calidad del viaje de las personas.

En el contexto local, estas desventajas hacen que algunos participantes elijan tomar autobuses, ya que no solo piensan en lo económico o en la rapidez del transporte sino en su seguridad y comodidad. Algunos prefieren consumir más tiempo en viajar pero sentirse mejor. En este sentido, el viajar no solo implica llegar rápidamente a destino, sino que la gente pueda trasladarse lo más tranquilamente posible, y disminuir las fuentes de estrés que pueden generar un transporte público. Y, por qué no, como plantean Morris y Guerra (2015), disfrutar de un paisaje que brinda luz y aire. Puede ser que estas características primen, porque las distancias que recorre el subterráneo de Buenos Aires son relativamente pequeñas. A diferencias de otras ciudades del mundo, el subterráneo no llega ni a los suburbios ni recorre toda la extensión de la ciudad.

La inversión del Estado en ampliar la red de subterráneos va a mejorar el viaje interurbano y el tráfico en la ciudad, y promoverá una mayor equidad social, alcanzando zonas de la ciudad invisibilizadas (Diab et al., 2017; Shuffield, 2017; Siu \& Zhao, 2013). Sin embargo, deberán encontrar la 
manera para aumentar la frecuencia del transporte, para controlar el aglomeramiento, aumentar la calidad del servicio y disminuir los factores asociados al estrés de viajar.

Como política del gobierno, se está invirtiendo en la ampliación del sistema de subterráneo, en la renovación de los vagones y en la subvención del servicio. Sin embargo, el crecimiento de esta red es históricamente muy lento y no atiende a ciertas problemáticas locales como la seguridad de los pasajeros. Como decían Légal et al. (2016), viajar es una práctica cotidiana de las personas, y se vuelve indispensable en una ciudad tan grande y cosmopolita como Buenos Aires. Esta cotidianeidad hace que las personas naturalicen el malestar y el estrés. Sin embargo, esto no implica que no las afecte negativamente. Si se comprende el grado en que puede mejorar la calidad de vida de las personas, se podrá comprender la urgencia en mejorar el servicio de transporte público.

Este estudio tiene ciertas limitaciones a nivel muestral, como el sesgo en el nivel educativo de los participantes y la percepción de clase social. Además, por las características cualitativas del estudio, no se aplicó una escala que indague cuantitativamente el bienestar. En próximas investigaciones, se planificará una investigación cuantitativa que contemple estas limitaciones y pueda explorar nuevas variables como la violencia en el subte y la presencia o ausencia de comportamientos cooperativos.

\section{REFERENCIAS}

Bell, M. G. H \& Iida, Y. (1997). Transportation Network Analysis. Nueva York: Wiley. https://doi.org/10.1002/9781118903032

Centro de Estudios Nuevo Milenio (2016). Encuesta sobre el transporte público de Capital Federal. Recuperado de http://www.centronuevomilenio.org/nuevo-milenio.

Corbin, J. (2017). Grounded theory. The Journal of Positive Psychology, 12(3), 301-302. https://doi.org/10.1080/17439760.2016.1262614

Cox, T., Houdmont, J., \& Griffiths, A. (2006). Rail passenger crowding, stress, health and safety in Britain. Transportation Research Part A: Policy and Practice, 4O(3), 244-258. https://doi.org/10.1016/j.tra.2005.07.001

Diab, E., van Lierop, D., \& El-Geneidy, A. (2017). Recommending transit: Disentangling users' willingness to recommend transit and their intended continued use. Travel Behaviour and Society, 6, 1-9. https://doi.org/10.1016/j.tbs.2016.03.001

Domínguez Sánchez-Pinilla, M. \& Davila Legerén, A. (2008 ). La práctica conversacional del grupo de discusión: jóvenes, ciudadanía y nuevos derechos. En A. Gordo \& A. Serrano (Eds.), Estrategias y prácticas cualitativas de investigación social (pp. 97-125). Madrid: Pearson Educación.

Ettema, D., Gärling, T., Eriksson, L., Friman, M., Olsson, L. E., \& Fujii, S. (2011). Satisfaction with travel and subjective well-being: Development and test of a measurement tool. Transportation Research Part F: Traffic Psychology and Behaviour, 14(3), 167-175. https://doi.org/10.1016/j.trf.2010.11.002

Evans, G. W., \& Wener, R. E. (2007). Crowding and personal space invasion on the train: Please don't make me sit in the middle. Journal of Environmental Psychology, 27(1), 90-94. https://doi.org/10.1016/j.jenvp.2006.10.002

Fernández Chaves, F. (2002). El análisis de contenido como ayuda metodológica para la investigación. Revista de Ciencias Sociales, II(96), 35-53.

Friman, M. (2010). Affective dimensions of the waiting experience. Transportation Research Part F: Traffic Psychology and Behaviour, 13(3), 197-205. https://doi.org/10.1016/j.trf.2010.04.006

Girabsas (septiembre, 2016) ¿Cuál es la sensación de los pasajeros del subte sobre el servicio? Recuperado de http://www.girabsas.com/nota/2016-9-12--cual-es-la-sensacion-de-los-pasajeros-del-subte-sobreel-servicio.

Gobierno de la Ciudad de Buenos Aires (2017). Encuesta Coincidental Subte. Recuperado de http://lppargentina.org.ar/subtedata/wp-content/uploads/2018/02/encuentas-coincidentalesseptiembre-2017.pdf.

Heritage, J. (2004). Conversation analysis and institutional talk: Analyzing data. En D. Silverman (Ed.), Qualitative research: Theory, method, and practice (pp. 222-245). Thousand Oaks, CA, EE. UU.: Sage.

INFOBAE (3 de enero de 2014). A un año del traspaso a Ciudad, aumentaron las quejas en subte. Recuperado de http://www.infobae.com/2014/01/03/1534669-a-un-ano-del-traspaso-ciudadaumentaron-las-quejas-subte.

Jain, S., Aggarwal, P., Kumar, P., Singhal, S., \& Sharma, P. (2014). Identifying public preferences using multi-criteria decision making for assessing the shift of urban commuters from private to public 
transport: A case study of Delhi. Transportation Research Part F: Traffic Psychology and Behaviour, 24, 60-70. https://doi.org/10.1016/j.trf.2014.03.007

Légal, J.-B., Meyer, T., Csillik, A., \& Nicolas, P.-A. (2016). Goal priming, public transportation habit and travel mode selection: The moderating role of trait mindfulness. Transportation Research Part F: Traffic Psychology and Behaviour, 38, 47-54. https://doi.org/10.1016/j.trf.2016.01.003

Li, S., Yang, J., Qin, P., \& Chonabayashi, S. (2016). Wheels of fortune: Subway expansion and property values in beijing. Journal of Regional Science, 56(5), 792-813. https://doi.org/10.1111/jors.12284

Maister, D. (1985). The psychology of waiting lines. Recuperado de http://davidmaister.com/articles/thepsychology-of-waiting-lines/.

Mohd Mahudin, N. D., Cox, T., \& Griffiths, A. (2012). Measuring rail passenger crowding: Scale development and psychometric properties. Transportation Research Part F: Traffic Psychology and Behaviour, 15(1), 38-51. https://doi.org/10.1016/j.trf.2011.11.006

Morris, E. A., \& Guerra, E. (2015). Are we there yet? Trip duration and mood during travel. Transportation Research Part F: Traffic Psychology and Behaviour, 33, 38-47. https://doi.org/10.1016/j.trf.2015.06.003

Muñoz Justicia, J. (2005). Análisis cualitativo de datos textuales con ATLAS TI. 5. Barcelona: Universidad Autónoma de Barcelona.

Nesprias, A. J. (2016). Hundirse en la ciudad. Pensar un subte para Buenos Aires (1887-1911). Instituto de Arte Americano e investigaciones, 108, 1-35.

Novillo, P. (11 de mayo de 2015). El subte suma pasajeros y quejas porque se viaja mal. Clarin. Recuperado de http://www.clarin.com/ciudades/subte-mas_pasajeros-pocos_coches_nuevosse_viaja_peor_0_1355264470.html.

Onwuegbuzie, A. J., Dickinson, W. B., Leech, N. L. \& Zoran, A. G. (2011). Un marco cualitativo para la recolección y análisis de datos en la investigación basada en grupos focales. Paradigmas, 3, 127-157.

Shen, W., Xiao, W., \& Wang, X. (2016). Passenger satisfaction evaluation model for Urban rail transit: A structural equation modeling based on partial least squares. Transport Policy, 46, 20-31. https://doi.org/10.1016/j.tranpol.2015.10.006

Shuffield, J. (2017). The Subway as Intermediary Public Space. Recuperado de http://www.urbanresidue.com/theory/subway.html.

Singh, D. Z. (2013). El subte como artefacto cultural (Buenos Aires, 1886-1944). La historia cultural como aporte a lose studios de las movilidades urbanas. Revista Transporte y Territorio, 9, 173-200.

Singh, D. Z. (2014). Meaningful mobilities: the experience of underground travel in the Buenos Aires Subte, 1913-1944. The Journal of Transport History, 35(1), 97-113. https://doi.org/10.7227/TJTH.35.1.7

Siu, K. \& Zhao, T. (2013). City Spaces and Human Relations in Hong Kong's Mass Transit Railway: From Circulation to Everyday Life. Journal of Human Behavior in the Social Environment, 23(5), 675-688. https://doi.org/10.1080/10911359.2013.788462

Strauss, A. \& Corbin, J. (2002). Bases de la investigación cualitativa. Técnicas y procedimientos para desarrollar la teoria fundamentada. Colombia: Editorial Universidad de Antioquía.

Subterráneos de Buenos Aires Sociedad del Estado (2017). Subte. Recuperado de http://www.buenosaires.gob.ar/subte.

${ }^{i}$ Movimiento de protesta social. 


\section{ANEXO 1}

Citas de los comentarios de los participantes.

1-a Yo lo uso por la celeridad, la comodidad que tiene y aún con sus limitaciones de sus distintas "ramificaciones", pero es muy ágil, es muy rápido.

1-b Si en mi caso también, es el tiempo en general, sobre todo en Capital, Microcentro. Con el colectivo tenés una hora una hora y media y con el subte en 40 minutos estás.

1-c Y yo, rapidez y conocimiento. No solo lo uso acá en Argentina, sino en todos lados. Al colectivo tengo que estudiarlo y me puedo confundir, mientras que con el subte desde chico, acá y en muchos países del mundo ya lo conozco. Quizás camino 8 cuadras más, que le voy a hacer.

1-d Y quizás también hoy en día a cómo están los precios, o sea los boletos, tal vez me conviene más tomarme el subte, hacer las combinaciones, con el boleto de lo que sale me tomaría dos colectivo. Por eso lo tomo, un poco por economía del estudiante.

2-a No, era excelente y fue inaugurado por nuestro presidente Victorino de la Plaza. Hay una foto del día. Pero suerte que ahora estamos mejorando la línea de subte. Antes no existía la $\mathrm{H}$, en los últimos años se inauguró la $\mathrm{H}$, se extendieron las líneas. Yo para venir aquí tome el subte, y el subte de la $\mathrm{A}$ antigua a este, no hay punto de comparación.

2-b La ventaja es que son más limpios visualmente también, no hay tanta publicidad como era antes no permiten que peguen carteles.

2-c Si la gente, yo creo que socialmente, yo creo que también uno está más atento y solidario en eso ¿no? Si ves algo raro haces miradas o decís algo para que no puedan robar.

3-a Según en mi caso por el horario en el que lo tomo, que por lo general son horarios pico de la mañana, a veces también lo tomo al medio día, y tengo que dejar pasar unidades según donde lo tome, o sea no me puedo subir, según la estación, si lo tomo en cabecera si me subo.

3-b Ahí tenés otra desventaja, no tener vista para afuera, siempre tenés que encontrarte en un lugar con otras miradas o estas con el celular o un libro, tenés que estar mirando, es bastante incomodo a veces.

3-c Para mi hay gente muy sucia y después no sé cómo harán la parte de mantenimiento.

4-a Yo había escuchado un proyecto que querían llevarlo a 2 minutos, pero no sé si se puede aplicar a todas las líneas por este tema que decís de infraestructuras, las nuevas por ahí ya lo están pensando tal vez tienen más alternativa y demás, pero acá por lo menos este, el C no tiene mucha.

4-c Y también el tema de las escaleras. En las horas pico la evacuación de los andenes...las escaleras son demasiado chicas y tampoco la gente se ayuda para utilizar la escalera mecánica. En Europa, los que quieran quedarse en el escalón que sube se pone a la derecha y los que quieran montarse sobre los escalones que suben utilizan la izquierda. En la Argentina algunos van montando y luego le toca a una persona que no y se queda estática en la izquierda.

4-d Después en las estaciones de las líneas que quedaron sin renovar, los andenes son muy angostos para la cantidad de gente que hay. Hay menos de un metro entre el inicio del andén y la línea amarilla en la que uno tiene que esperar

4-e Si hay dos personas le queda un espacio así, una persona chiquita entra pero alguien grande no se sienta porque le da vergüenza, porque sabe que no va a entrar, no están preparados para nuestra contextura

4-g (...) que cuando a veces se da que esta demorado o que se yo, te cambia el humor, vos venís corriendo y te encontrás con el cartel que dice tren con demora y no te saben decir de cuánto es la demora y otro cuando estas dentro del subte y dicen, "la formación no sale" eso a mí me saca. Y se genera una cuestión de incertidumbre, nadie sabe nada, te acercas a boletería y preguntas en terminales como en San Pedrito o Plaza de Mayo que estas cuatro o 5 personas que están esperando formación para subir y no tienen ni idea, no se quien tiene que dar aviso o quien debe ser el gran Dios que avisa, pero no es un desastre.

4-h No, no te hablo de la periferia tampoco ¿eh? Vos tenés el subte donde en realidad está todo lo focal de la ciudad, muchas universidades, los lugares gubernamentales, ahí tenés subte por todos lados, pero ya está. Para llegar al subte si vivís más lejos, tenés que tomarte un tren o un colectivo. Yo para venir acá tuve que tomarme un tren y después el subte. O sea es como acotado. Estaría bueno que abarque más, no te digo que llegue a provincia porque sería como utópico, pero sí que abarque más Capital.

5-a Siii! Si me siento mucho más desprotegida. No sé por qué, será tal vez que en el colectivo está el chofer y lo para antes que pase algo y en el subte todo sigue, es como si, es una sensación difícil de explicar pero me siento desprotegida en el subte.

5-b Vos estas en un lugar donde sabes que hay alta posibilidad que alguno de los que está ahí sea un chorro (e.g., ladrón) (...). O sea, a mi parece que aunque uno no lo haga consciente, inconscientemente esa inquietud, ese miedo, esa angustia está. Me van a afanar (i.e., robar) o no me van a afanar. A mí me afanaron tres veces en el subte y en un caso ni me di cuenta (se ríe), me di cuenta cuando me agarré lo bolsillo.

$6 \quad$ El subte por sobre el coche. Porque el coche tiene el problema de dónde lo estacionás, que te cobran un disparate en cada estacionamiento y que en los horarios pico, de salida y entrada al Centro, tenés manifestaciones, embotellamiento de tránsito. Yo prefiero el subte antes que el coche, aunque tenga coche.

Recibido 20-05-2019 | Aceptado 12-08-2019

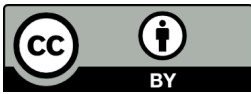

Este trabajo se encuentra bajo una Licencia Creative Commons Atribución 4.0 Internacional que permite a terceros utilizar lo publicado siempre que se dé el crédito pertinente a los autores y a Psicodebate 